Supporting Information

\title{
Eu(III)-Chelated Polymeric Hybrid Nanoplatform for Luminescence Resonance Energy Transfer (LRET)-Based Real-Time Monitoring of Organic Cargo-Release
}

\author{
Wonjeong Yu, Yeojin Kim, Yoojin Jang, and Sang-Min Lee* \\ Department of Chemistry, The Catholic University of Korea, Bucheon, Gyeonggi-do 14662, Korea. \\ *E-mail: smlee120@ catholic.ac.kr
}

\section{Experimental Section}

Materials. $N, N, N$ ',$N$ ',,$N$ ''-Pentamethyldiethylenetriamine (PMDETA, 99\%), ethyl $\alpha$-bromoisobutyrate (EBiB, 98\%), copper (I) bromide (98\%), tetrahydrofuran (THF, anhydrous) Nile Blue A, and Europium(III) chloride hexahydrate ( $\geq 99.9 \%$, trace metals basis) were purchased from Aldrich Chemical Co. (Milwaukee, WI) and used without further purification. tert-butyl acrylate ( $t \mathrm{BA}, 98 \%)$ was stirred over $\mathrm{CaH}_{2}$ under nitrogen and fractionated by vacuum transfer right before use. Poly(ethylene glycol) methyl ether methacrylate (PEGMA, $\mathrm{M}_{\mathrm{n}}=500 \mathrm{~g} / \mathrm{mol}$ ) were purified by passing through an alkaline aluminum oxide column to remove the radical inhibitor. Trifluoroacetic acid (TFA, 99\%), sodium nitrate (98\%), hydrochloric acid (35\%), and all other reagents including typical organic solvents were purchased from Samchun Chemical Co. (Pyeongtaek, Korea) and used as received. Regenerated Cellulose (RC) dialysis membranes were purchased from Spectrum Laboratories (Thermo scientific, Waltham, MA). Deionized (DI) water was obtained from Human Power I ${ }^{+}$Scholar-UV (Human Corporation, Seoul, Korea) (18.2 M $\Omega$ cm resistivity).

Measurements. Instrumental analyses were carried out at the Cooperative Center for Research Facilities of the Catholic University of Korea. Fourier-transformed nuclear magnetic resonance (NMR) spectroscopy was carried out on an AVANCE III $300 \mathrm{MHz}$ spectrometer. Chemical shifts of ${ }^{1} \mathrm{H}$ NMR spectra are reported in ppm against residual solvent resonance as the internal standard $\left(\mathrm{CDCl}_{3}=7.27 \mathrm{ppm}, \mathrm{D}_{2} \mathrm{O}=4.8\right.$ $\mathrm{ppm}$ ). The samples were prepared by dissolving $5 \pm 1 \mathrm{mg}$ in $0.7 \mathrm{~mL}$ of deuterated solvents in a $5 \mathrm{~mm} \mathrm{NMR}$ tube. Ultraviolet-visible (UV-vis) absorption spectra were obtained using a Lambda 35 spectrophotometer (Perkin Elmer, Waltham, MA). Fluorescence emission/excitation spectra were obtained on a FluoroMate FS-2 fluorometer (Scinco Co., Ltd. Seoul, Korea) at a scan speed of $100 \mathrm{~nm} / \mathrm{min}\left(\lambda_{\mathrm{Ex}}=395 \mathrm{~nm}, \lambda_{\mathrm{Em}}=617\right.$ $\mathrm{nm}$, slit width $=2.5 \mathrm{~nm}$ for $\mathrm{Eu}(\mathrm{III})$ luminescence; $\lambda_{\mathrm{Ex}}=635 \mathrm{~nm}, \lambda_{\mathrm{Em}}=674 \mathrm{~nm}$, slit width $=2.5 \mathrm{~nm}$ for NB fluorescence).

DLS and zeta $(\zeta)$ potential measurements were carried out with Zetasizer Nano ZS (Malvern Instruments, Malvern, UK). The size distribution of the nanoparticles was obtained by the non-negative least-squares analysis. The $\zeta$-potential of each sample was calculated from the electrophoretic mobility values using the Smoluchowski equation. Unless noted otherwise, all samples were dispersed in $10 \mathrm{mM}$ phosphate buffer ( $\mathrm{pH}$ 7.4) for measurements. The data reported represent an average of 10 measurements with five scans each. 
Gel-permeation chromatograph (GPC) measurements were carried out with Agilent 1260 Infinity LC system equipped with Shodex KF-803L GPC column connected to an Agilent 1260 RI detector. HPLCgrade THF was used as an eluent at a flow rate of $0.5 \mathrm{~mL} / \mathrm{min}$ and the instrument was calibrated using polystyrene standards (Aldrich, 15 standards, 760-1,800,000 Daltons) by OpenLAB CDS ChemStation (ver. A.2.3.57) software. Aqueous phase size-exclusion chromatography (SEC) was carried out on an Agilent 1260 Infinity II GPC System (three Shodex polyhydroxymethacrylate gel columns OHpak SB-G guard column, SB-802.5HQ, SB-803HQ, and SB-804HQ columns in series) connected to an Agilent 1260 MultiDetector Suite (MDS) with RI and LS detectors using $10 \mathrm{mM}$ phosphate-buffered ( $\mathrm{pH} \mathrm{7.4,} 140 \mathrm{mM} \mathrm{NaNO}_{3}$ ) aqueous solution as an eluent at a flow rate of $0.5 \mathrm{~mL} / \mathrm{min}$. The SEC instrument was calibrated with six different linear PEG/polyethylene oxide standards $(0.55,2,5,35,100$, and $600 \mathrm{kDa})$ and the apparent mass of each sample was automatically calculated by Agilent GPC/SEC software (ver. A.02.01).

Energy-filtering transmission electron microscope (EF-TEM) observation was carried out with a LIBRA 120 (Carl Zeiss, Oberkochen, Germany) with a beam voltage of $120 \mathrm{kV}$ and a slow-scan chargecoupled device at the National Instrumentation Center for Environmental Management in Seoul National University. The sample solution $(50 \mu \mathrm{L})$ was placed on a copper grid (200 mesh) for $30 \mathrm{sec}$, and the excess solution was blotted with filter paper. The grid was dried at room temperature for $24 \mathrm{~h}$. Eu(III)-chelated samples were observed without staining.

Synthesis of PAA- $b$-PEGMA Block-Copolymer. PAA- $b$-PEGMA block-copolymers was prepared using a modified literature procedure (Scheme S1). ${ }^{1}$ Briefly, $\mathrm{CuBr}(71.7 \mathrm{mg}, 0.5 \mathrm{mmol})$ were added to a 50-mL dry Schlenk flask equipped with a magnetic stir bar, which was then sealed with a rubber septum, degassed, and back-filled with nitrogen three times. Anhydrous acetone $(9 \mathrm{~mL})$, tert-BA $(10.95 \mathrm{~mL}, 75 \mathrm{mmol})$, and PMDETA $(105 \mu \mathrm{L}, 0.5 \mathrm{mmol})$ were subsequently added via syringe under inert condition. Next, the solution was stirred until the $\mathrm{Cu}$ complex was formed, which can be easily recognized by a color change from colorless/turbid to a greenish clear solution. After complex formation, ethyl $\alpha$-bromoisobutyrate (73.5 $\mu \mathrm{L}, 0.5 \mathrm{mmol}$ ) was added and degassed by three cycles of freeze-pump-thaw. Then, the flask was placed in an oil bath and polymerization was carried out at $60{ }^{\circ} \mathrm{C}$. After the predetermined reaction time, the polymerization was quenched by placing the reaction flask in an ice bath. After the evaporation of acetone under reduced pressure, the reaction product was purified by passing through a neutral alumina column with THF to remove the copper catalysts. The resulting product was precipitated with a 10 -fold excess of water $/ \mathrm{MeOH}(50 / 50 \mathrm{vol} / \mathrm{vol})$ solution. The precipitate was dried under a vacuum and obtained as a colorless product.

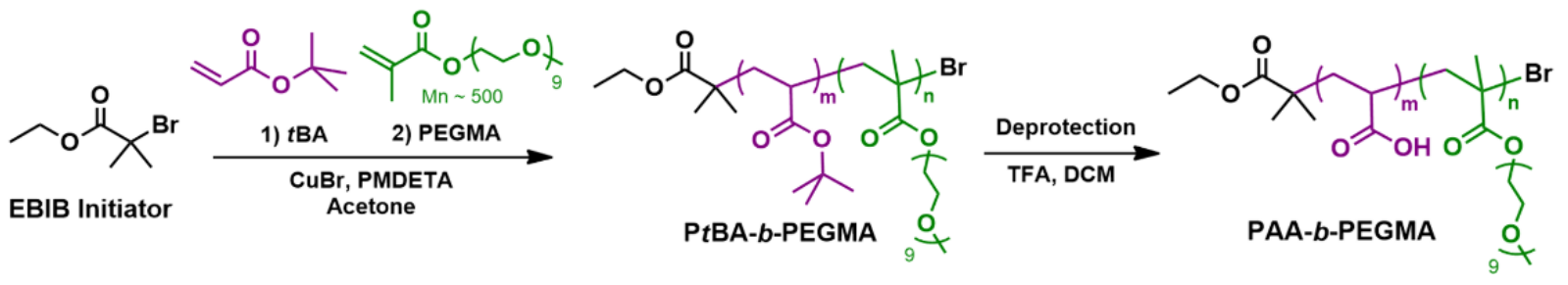

Scheme S1. Synthesis of PAA- $b$-PEGMA block-copolymers via atom-transfer radical polymerization.

For the polymerization of second block, bromo-poly $(t \mathrm{BA})\left(\mathrm{M}_{\mathrm{n}}=7629 \mathrm{~g} / \mathrm{mol}, 1.53 \mathrm{~g}, 0.2 \mathrm{mmol}\right)$, $\mathrm{CuBr}(29.0 \mathrm{mg}, 0.2 \mathrm{mmol})$, and anhydrous acetone $(9 \mathrm{~mL})$ were added to a 50 - $\mathrm{mL}$ dry Schlenk flask equipped with a magnetic stir bar. PEGMA ( $2 \mathrm{~g}, 4 \mathrm{mmol})$ and PMDETA (41.8 $\mu \mathrm{L}, 0.2$ mmol) were subsequently added via a purged syringe, which was then stirred until the $\mathrm{Cu}$ complex was formed. The 
mixture was degassed by three cycles of freeze-pump-thaw. Then, the flask was placed in an oil bath and polymerization was carried out at $60{ }^{\circ} \mathrm{C}$. After the predetermined reaction time, the polymerization was quenched by placing the reaction flask in an ice bath. After the evaporation of acetone under reduced pressure, the reaction product was purified by passing through a neutral alumina column with THF to remove the copper catalysts. The molecular weight of the resulting poly(tBA)- $b$-(PEGMA) was measured by gel permeation chromatography (GPC) (Figure S2B). For acid-mediated deprotection of tert-butyl groups in poly $(t \mathrm{BA})$, to a $100-\mathrm{mL}$ round-bottom flask equipped with a magnetic stir bar was added the polymers product $(3.46 \mathrm{~g})$, DCM $(25 \mathrm{~mL})$, and TFA $(5.11 \mathrm{~mL}, 99 \%)$, which was then sealed with a rubber septum and insert a needle on it, followed by stirring for $120 \mathrm{~h}$ at room temperature. After DCM was evaporated under vacuum, the crude product was neutralized with aqueous $\mathrm{NaOH}(10 \mathrm{~mL}, 15 \%)$ and dialyzed $(\mathrm{MWCO}=3500 \mathrm{Da})$ against D.I. water $(7 \times 1000 \mathrm{~mL})$ for 7 days with water change every $24 \mathrm{~h}$. After dialysis, the product was dried by lyophilization and obtained as a colorless product.
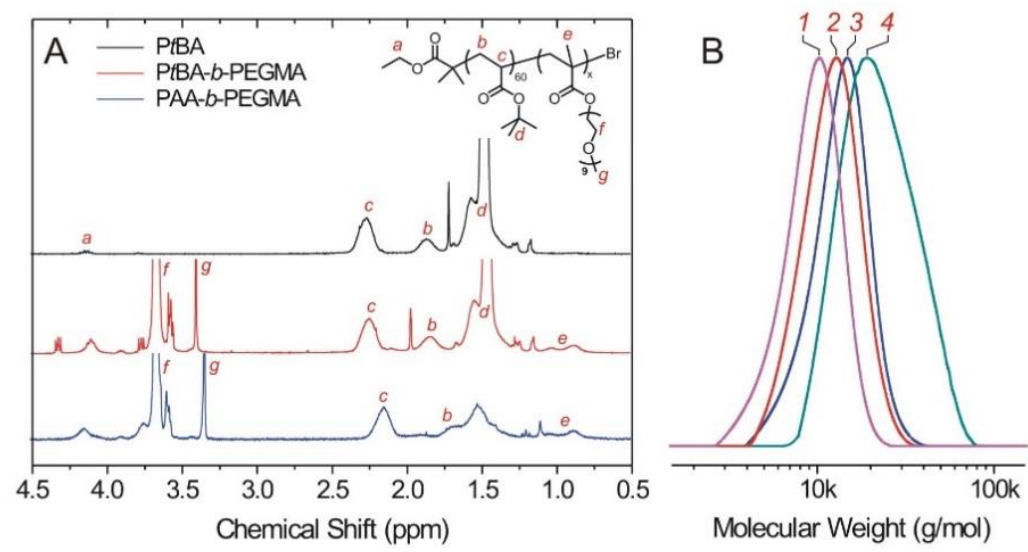

\begin{tabular}{cccc} 
No. & sample & $\begin{array}{c}\mathrm{M}_{\mathrm{n}} \\
(\mathrm{kDa})\end{array}$ & $\bigoplus_{\mathrm{M}}$ \\
1 & $\mathrm{P}(t \mathrm{BA})_{50}$ & 8.6 & 1.14 \\
2 & $\mathrm{P}(t \mathrm{BA})_{60}$ & 10.1 & 1.22 \\
3 & $\mathrm{P}(t \mathrm{BA})_{70}$ & 11.4 & 1.19 \\
4 & $\mathrm{P}(t \mathrm{BA})_{60}-b-$ & 15.3 & 1.41 \\
\hline
\end{tabular}

Figure S1. (A) The representative ${ }^{1} \mathrm{H}$ NMR spectra of PtBA, PtBA- $b$-PEGMA in $\mathrm{CDCl}_{3}$, and PAA- $b$ PEGMA in $\mathrm{D}_{2} \mathrm{O}$. (B) GPC traces of $\mathrm{P} t \mathrm{BA}$ and $\mathrm{P} t \mathrm{BA}-b$-PEGMA in THF (left). Detailed information of each polymer is listed in the table (right). The degree of polymerization of each polymer sample was determined independently by ${ }^{1} \mathrm{H}$ NMR integration.

Preparation of Eu(III)-Chelated Polymeric Nanoparticles (Eu-PNPs). The Eu(III)-chelated polymeric nanostructures were prepared using a modified literature procedure. ${ }^{2}$ To a 5 - $\mathrm{mL}$ glass vial containing the aqueous solution of $\mathrm{EuCl}_{3}(3.3 \mathrm{mg}, 9 \mu \mathrm{mol})$ was added the $\mathrm{pH}$-adjusted aqueous solution $(\mathrm{pH} 7.5,1.2 \mathrm{~mL})$ of PAA- $b$-PEGMA $\left(6.12 \mathrm{mg}, 0.155 \mu \mathrm{mol},[\mathrm{AA}] /\left[\mathrm{Eu}^{3+}\right]=4.0\right)$ incubated at room temperature for $24 \mathrm{~h}$. Then, the resulting transparent solution of Eu-PNPs were purified by Amicon Ultra centrifugal filter $(5 \mathrm{~mL}$, MWCO $10000 \mathrm{Da}$ ), followed by redispersion in aqueous solution. Identical results were also obtained with $\mathrm{Eu}\left(\mathrm{NO}_{3}\right)_{3}$ instead of $\mathrm{EuCl}_{3}$.

Determination of Eu(III) Quantity in Eu-PNPs. The amount of Eu(III) ions in Eu-PNPs was determined by UV-vis spectroscopy using a predetermined calibration curve. Briefly, the excess amount of free Eu(III) that was not associated to Eu-PNPs was separated with Amicon Ultra centrifugal filter (5 mL, MWCO 10 $\mathrm{kDa}$ ). Then, the quantity of free $\mathrm{Eu}(\mathrm{III})$ ions was determined by UV-Vis absorption, using the predetermined molar extinction coefficient $(\varepsilon)$ of $\mathrm{Eu}(\mathrm{III})$ ions $\left(\varepsilon=2.10 \mathrm{M}^{-1} \cdot \mathrm{cm}^{-1}\right.$ at $\left.\lambda_{\text {Max }}=394 \mathrm{~nm}\right)$. The amount of $\mathrm{Eu}(\mathrm{III})$ ions chelated in Eu-PNPs was relatively determined by comparing the amount of free $\mathrm{Eu}(\mathrm{III})$ and the initial Eu(III) introduced to the polymers (Figure S3). 


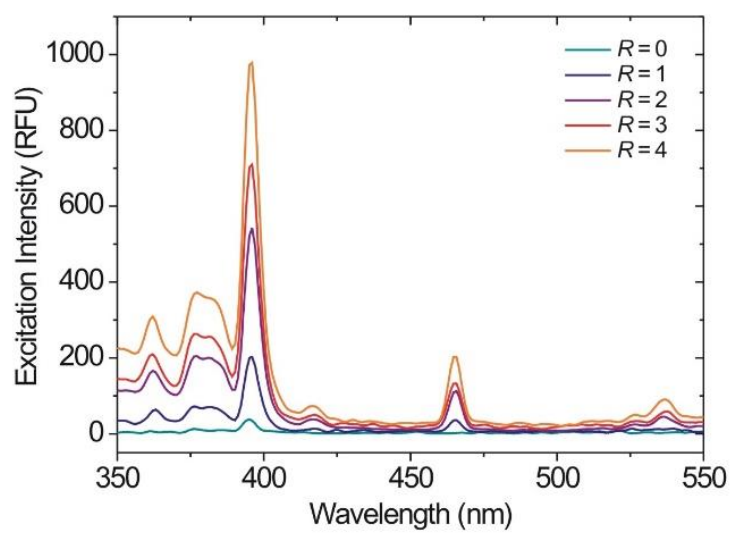

Figure S2. Luminescence excitation spectra of Eu-PNPs depending on the $R$ values.
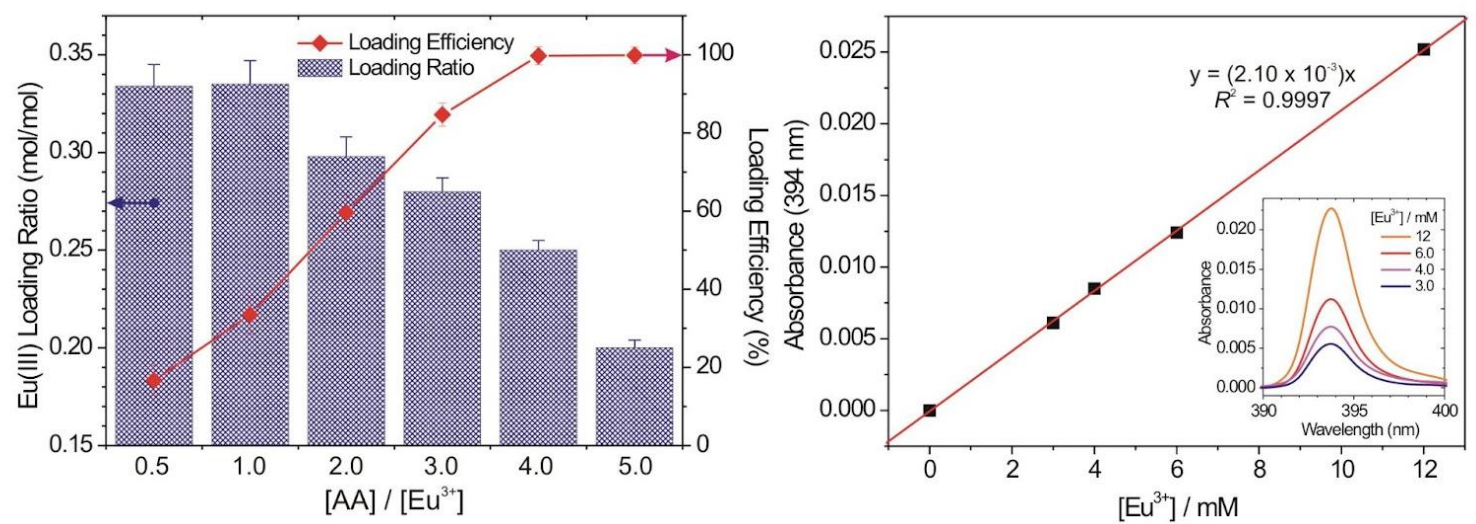

Figure S3. (A) Eu(III) binding ratio (bar graph) relative to the acrylate (AA) repeating units in PAA- $b$ PEGMA and the corresponding binding efficiency (red line). At a constant concentration of aqueous $\mathrm{Eu}(\mathrm{III})$, the polymer-dependent $\mathrm{Eu}(\mathrm{III})$ binding $\mathrm{mol} / \mathrm{mol}$ ratio in Eu-PNPs was measured by the absorbance of aqueous Eu(III) ion at $394 \mathrm{~nm}$. (B) Calibration curve of Eu(III) ion in aqueous solution. Inset shows the concentration-dependent absorption spectra of Eu(III) ions in aqueous solution.

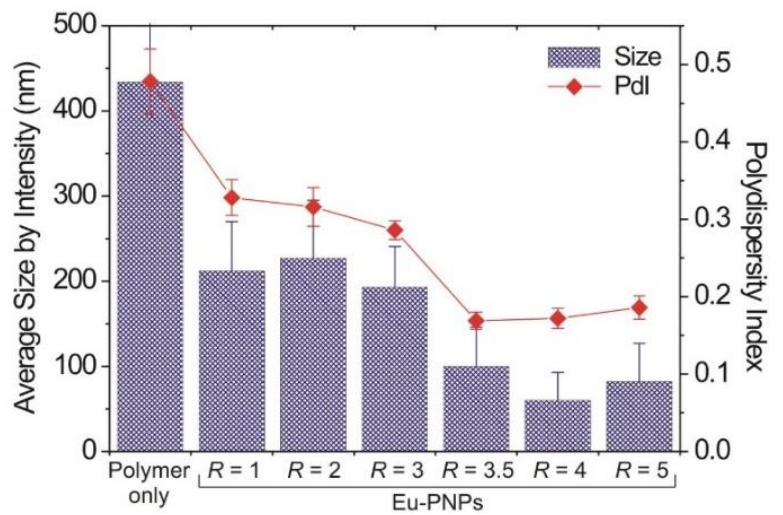

Figure S4. $R$-dependent average size of Eu-PNPs and the corresponding polydispersity index (PdI) measured by DLS. 


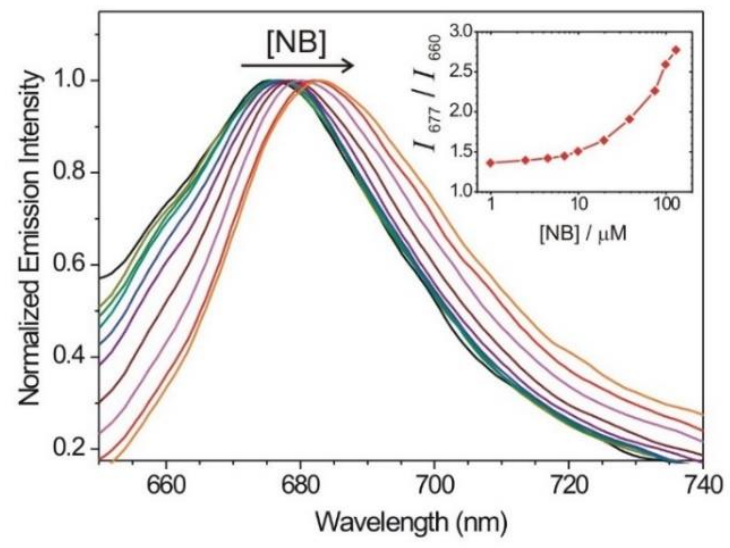

Figure S5. Normalized emission spectra of NB after polymer association, showing the aggregationdependent red-shift $\left(\lambda_{\mathrm{Ex}}=635 \mathrm{~nm}\right)$.
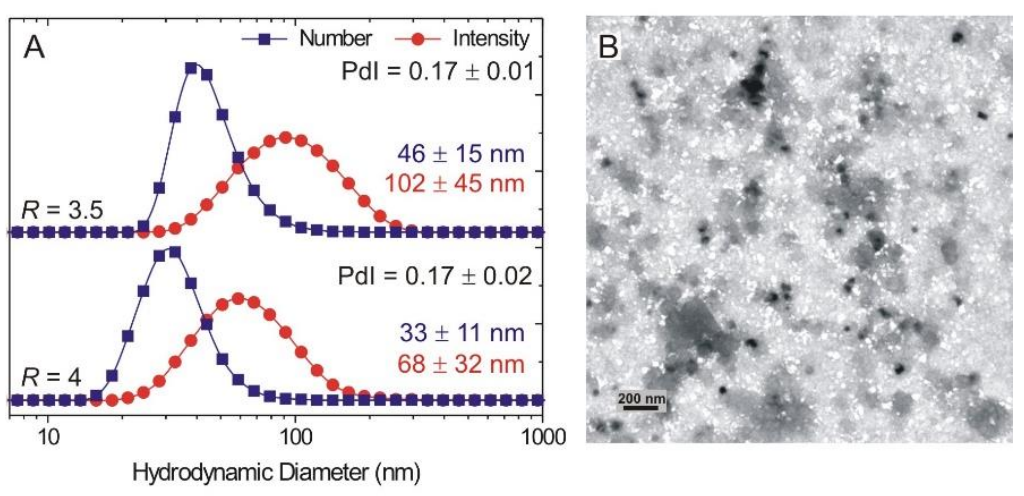

Figure S6. (A) DLS size distribution of Eu-PNPs/NB of $R=3.5$ and 4.0. (B) TEM image of Eu-PNPs/NB $(R=4.0)$.

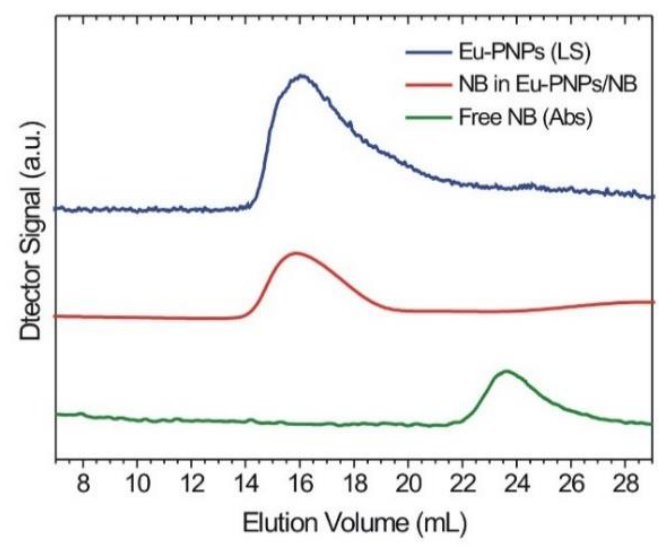

Figure S7. Aqueous-phase SEC traces of Eu-PNPs, NB in Eu-PNPs/NB, and free NB, showing a negligible amount of free NB observed after the formation of Eu-PNPs/NB $(R=4.0$ and [polym] $/ \mathrm{NB}=$ $10)$. 

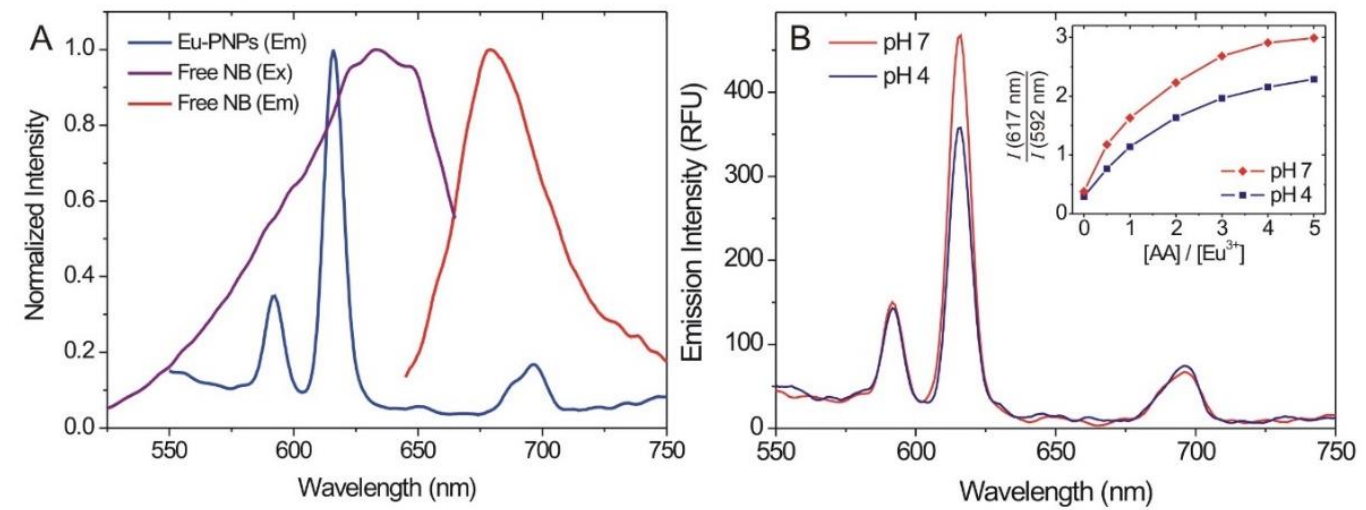

Figure S8. (A) Fluorescence excitation (Ex) and emission (Em) spectra of LRET donor (Eu-PNPs) and acceptor (Nile Blue, NB). $\lambda_{\mathrm{Ex}}=395 \mathrm{~nm}$ for Eu-PNPs; $\lambda_{\mathrm{Ex}}=635 \mathrm{~nm}$ and $\lambda_{\mathrm{Em}}=674 \mathrm{~nm}$ for NB emission and excitation spectrum, respectively. (B) pH-dependent luminescence emission spectra of Eu-PNPs with $R\left([\mathrm{AA}] /\left[\mathrm{Eu}^{3+}\right]\right)=4.0$. (Inset) $R$-dependent emission peak intensity ratio between $617 \mathrm{~nm}$ and $592 \mathrm{~nm}$ at different $\mathrm{pH}$ condition.
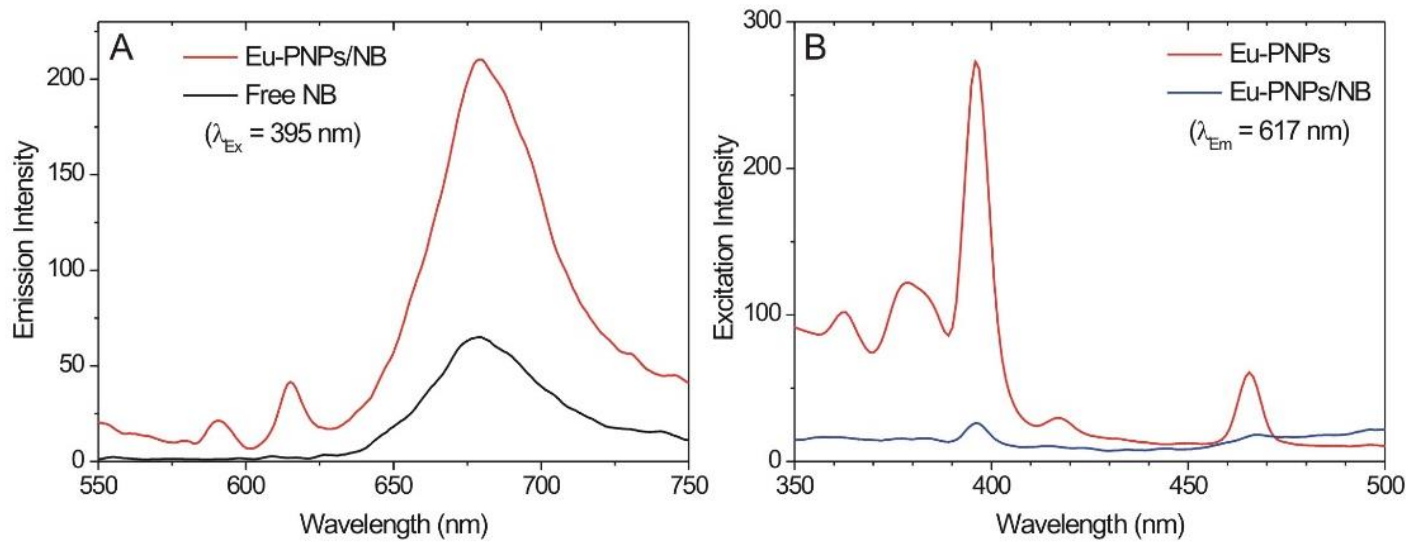

Figure S9. (A) Emission spectra of Eu-PNPs/NB (red line) and free NB (black line) in aqueous solution $\left(\lambda_{\mathrm{Ex}}=395 \mathrm{~nm}\right)$, showing LRET from Eu-PNPs $(617 \mathrm{~nm})$ to NB $(674 \mathrm{~nm})$. (B) Excitation spectra of Eu-PNPs (red line) and Eu-PNPs/NB (blue line) in aqueous solution $\left(\lambda_{\mathrm{Em}}=617 \mathrm{~nm}\right)$, showing the significant quenching of Eu-PNPs at $617 \mathrm{~nm}$ by NB incorporation. ${ }^{3}$

Evaluation of Colloidal Stability of Eu-PNPs against Human Serum Albumin (HSA). The protein stability of Eu-PNPs was evaluated using a modified literature procedure. ${ }^{2}$ An aliquot of Eu-PNPs solution was mixed with aqueous solution containing human serum albumin $(45 \mathrm{mg} / \mathrm{mL} \mathrm{HSA}, 150 \mathrm{mM} \mathrm{NaNO}$ ) in a capped 5-mL vial equipped with a magnetic stir bar and incubated at $37{ }^{\circ} \mathrm{C}$. The final concentration of copolymers was $3.825 \mathrm{mg} / \mathrm{mL}$. After the $24 \mathrm{~h}, 48 \mathrm{~h}$, and $72 \mathrm{~h}$ incubation, aliquots were withdrawn, diluted with isotonic solution ( $\mathrm{pH} 7.4$ and $150 \mathrm{mM} \mathrm{NaNO}_{3}$ ), and the solution turbidity of each sample was measured by UV-Vis absorption spectrometry in transmission mode.

NB Release Assay from Eu-PNPs/NB in HSA Solution. NB release from Eu-PNPs/NB was evaluated by the recovery of LRET using a modified literature procedure. ${ }^{4}$ For time-dependent monitoring of LRET recovery, solution of Eu-PNPs/NB containing HSA $(6.3 \mathrm{mg} / \mathrm{mL})$ in $10 \mathrm{mM}$ phosphate buffer $(\mathrm{pH} 7.4$ and 
$140 \mathrm{mM} \mathrm{NaNO}$ ) was incubated at $37{ }^{\circ} \mathrm{C}$ in a $1 \mathrm{~mL}$ Quartz fluorescence cuvette with magnetic stirring. The time-dependent luminescence/fluorescence emissions from Eu(III) complex (617 nm) and NB (674 $\mathrm{nm})$ were monitored for $72 \mathrm{~h}$. Afterward, EDTA equivalent to $\mathrm{Eu}(\mathrm{III})(\geq 4.5 \mathrm{mM})$ in Eu-PNPs/NB was added to completely deplete the $\mathrm{Eu}(\mathrm{III})$ ions from the nanoparticles and the final $I_{674} / I_{617}$ value was obtained to estimate the $100 \%$ release value. The extent of NB release was then determined by comparing the $I_{674} / I_{617}$ values of each time point to that of EDTA-treated sample (Figure S9).

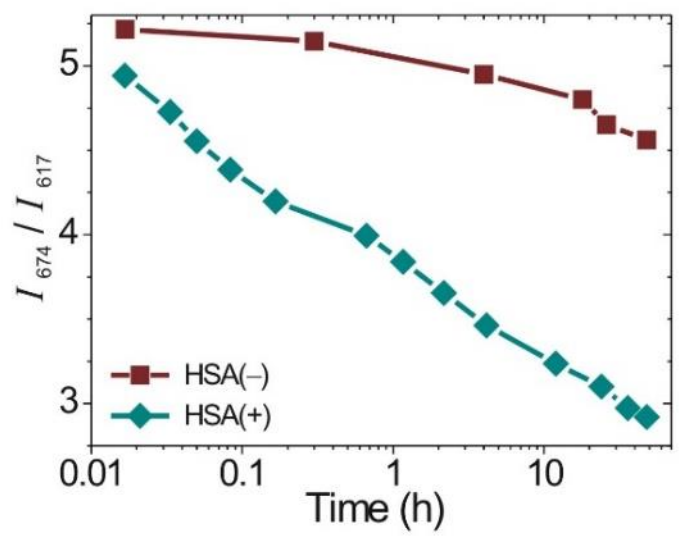

Figure S10. Plot of luminescence emission intensity ratio at $674 \mathrm{~nm}$ and $617 \mathrm{~nm}$ from Eu-PNPs/NB as a function of incubation time at $37^{\circ} \mathrm{C}$ in HSA solution.

\section{References.}

1. Davis, K. A.; Matyjaszewski, K., Atom Transfer Radical Polymerization of tert-Butyl Acrylate and Preparation of Block Copolymers. Macromolecules 2000, 33, 4039-4047.

2. Jeong, Y.-H.; Shin, H.-W.; Kwon, J.-Y.; Lee, S.-M., Cisplatin-Encapsulated Polymeric Nanoparticles with Molecular Geometry-Regulated Colloidal Properties and Controlled Drug Release. ACS Appl. Mater. Interfaces 2018, 10, 23617-23629.

3. Li, Q.; Zhang, J.; Sun, W.; Yu, J.; Wu, C.; Qin, W.; Chiu, D. T., Europium-Complex-Grafted Polymer Dots for Amplified Quenching and Cellular Imaging Applications. Langmuir 2014, 30, 8607-8614.

4. Lee, S.-M., Labile Incorporation of Cholesterol-Terminated Poly(Acrylic Acid) for the Facile Surface-Modification of Lipid Vesicles. Langmuir 2017, 33, 6751-6759. 